(partial) correlations showed significant associations between whole blood serotonin and BMI $(r=-0.284, p=0.021)$ and waist circumference $(r=-0.325, p=0.008)$. The correlational nature of these analyses do not enable conclusions to be drawn as to cause and effect but suggest an interplay between obesity and lower whole blood serotonin levels.

Funding This analysis has been funded by the HRB (Ireland) Health Research Centre.

\section{P2-290 SOCIOECONOMIC STATUS AND MORTALITY AMONG PEOPLE WITH DIABETES MELLITUS IN LATVIA}

doi:10.1136/jech.2011.142976k.23

${ }^{1}$ I Strele, ${ }^{* 1,2} \mathrm{~L}$ Golosujeva, ${ }^{1,2} \mathrm{~S}$ Rozite, ${ }^{1} \mathrm{G}$ Brigis, ${ }^{3} \mathrm{~V}$ Pirags. ${ }^{1}$ Riga Stradins University, Riga, Latvia; ${ }^{2}$ Centre of Health Economics, Riga, Latvia; ${ }^{3}$ University of Latvia, Riga, Latvia

Introduction The chronic course of the disease and regular need for healthcare suggests that people with diabetes mellitus might be more affected by the socioeconomic inequalities in health. We assessed whether socioeconomic differences exist in mortality of diabetes patients in Latvia.

Methods The study population consisted of 4179 diabetes patients (1339 men and 2840 women) participating in the Latvian diabetes survey in 2000. All deaths $(n=1771)$ during a subsequent ten-year period and causes of death were identified through the Causes of Death Data Base. The Cox model was used to test associations (HR ( $95 \% \mathrm{CI}$ ) presented) between education, income and residence (rural/urban) and mortality, adjusted for age, duration of diabetes, glycaemia, and blood pressure.

Results The primary education, compared to the higher education, was associated with an increase in all-cause, cardiovascular mortality and death from diabetes in both men (1.45 (1.16-1.82); 1.45 (1.07-1.95); 2.33 (1.23-4.40), respectively) and women (1.91 (1.53-2.38); 1.72 (1.31-2.25); 2.37 (1.25-4.48), respectively). The lowest income tertile, compared to the highest, was associated with higher all-cause mortality in both men (1.42 (1.14-1.77)) and women (1.37 (1.14-1.65)), as well as with higher cardiovascular mortality in men (1.44 (1.06-1.94)). Rural residence was associated with lower cardiovascular mortality in men $(0.70(0.50-0.98))$. The strength of associations did not change substantially when smoking, alcohol consumption, or parameters of diabetes care were included in the model.

Conclusion Overall, diabetes patients with poorer education and lower income experienced higher mortality. These inequalities were not explained by behavioural factors or diabetes care.

\section{P2-291 STRUCTURAL AND FUNCTIONAL MEASURES OF SOCIAL SUPPORT, SOCIOECONOMIC POSITION AND MORTALITY. THE BRITISH WHITEHALL II STUDY}

doi:10.1136/jech.2011.142976k.24

${ }^{1} \mathrm{~S}$ Stringhini, ${ }^{2} \mathrm{~L}$ Berkman, ${ }^{1} \mathrm{~A}$ Dugravot, ${ }^{3} \mathrm{~J}$ E Ferrie, ${ }^{3} \mathrm{M}$ Marmot, ${ }^{3} \mathrm{M}$ Kivimaki, ${ }^{1,3}$ A Singh-Manoux. ${ }^{1}$ INSERM U1018, Paris, France, ${ }^{2}$ Harvard school of Public Health, Boston, USA; ${ }^{3}$ University College London, London, UK

Introduction Social relationships are associated with socioeconomic position (SEP) and with mortality, but it is unclear to what extent the socioeconomic gradient in mortality is attributable to differences in social relations between socioeconomic groups. In this study, we examine the contribution of different measures of social support to the socioeconomic gradient in all-cause and cause-specific mortality.
Methods Analyses are based on 9333 participants from the British Whitehall II study, aged 35-55 years at baseline (1985). SEP was civil service employment grade at baseline and social support was assessed 3 times over a follow-up for mortality of 24.4 years.

Results Measures of social support were socially patterned, but the pattern differed by gender. In men, social isolation and marital status were predictors of all-cause $(\mathrm{HR}=1.27,95 \%$ CI 1.07 to 1.52 for low network score and $\mathrm{HR}=1.77$; $95 \%$ CI 1.45 to 2.16 for not being married) and cardiovascular mortality $(\mathrm{HR}=1.85$; 95\% CI 1.36 to 2.51 for low network score and $\mathrm{HR}=2.69$; $95 \% \mathrm{CI}$ 1.95 to 3.71 for not being married). These associations were partially attenuated after adjustment for self-rated health. Men in the lowest SEP had an higher mortality risk than those in the highest $(\mathrm{HR}=1.59 ; 95 \% \mathrm{CI}=1.21$ to 2.08 for all-cause and $\mathrm{HR}=2.48$; 95\% CI 1.55 to 3.92 for cardiovascular mortality). Network score and marital status attenuated these associations by $27 \%(95 \% \mathrm{CI}$ $14 \%$ to $43 \%$ ) and $29 \%$ ( $95 \%$ CI $17 \%$ to $52 \%$ ), respectively. In women, there was no consistent association between social support and mortality.

Conclusions The associations of social support with SEP and with mortality differ by gender. In men, social isolation and in particular not being married are important risk factors for mortality and are also likely to contribute to social inequalities in health.

\section{P2-292 TOTAL SERUM CHOLESTEROL AND CANCER INCIDENCE IN THE METABOLIC SYNDROME AND CANCER PROJECT (ME-CAN)}

doi:10.1136/jech.2011.142976k.25

${ }^{1} \mathrm{~S}$ Strohmaier, ${ }^{* 2,3} \mathrm{~T}^{\mathrm{T}}$ Bjørge, ${ }^{4} \mathrm{~J}$ Manjer, ${ }^{5} \mathrm{H}$ Concin, ${ }^{6} \mathrm{G}$ Nagel, ${ }^{7} \mathrm{~T}$ Stocks, ${ }^{7} \mathrm{P}$ Stattin ${ }^{1} \mathrm{H}$ Ulmer. ${ }^{1}$ Department of Medical Statistics, Informatics and Health Economics, Innsbruck, Austria; ${ }^{2}$ Department of Public Health and Primary Health Care, Bergen, Norway; ${ }^{3}$ Norwegian Institute of Public Health, Oslo, Norway; ${ }^{4}$ Department of Surgery, Malmö, Sweden; ${ }^{5}$ Agency for Preventive and Social Medicine, Bregenz, Austria; ${ }^{6}$ Institute of Epidemiology, Ulm, Germany; ${ }^{7}$ Department of Surgical and Perioperative Sciences, Umeå, Sweden

Introduction The relationship between serum cholesterol and cancer risk remains controversial. We examined the association between total serum cholesterol and cancer incidence in the Metabolic Syndrome and Cancer Project (Me-Can).

Methods Me-Can consists of seven cohorts from Norway, Austria, and Sweden including 289273 male and 288057 female participants prospectively followed up for cancer incidence $(n=39004)$ for a mean follow-up of 11.7 years. We used Cox regression models with age as the underlying time metric to calculate HRs and their $95 \%$ CIs for $1 \mathrm{mmol} / \mathrm{l}$ increment of total cholesterol levels adjusting for age at first measurement, body mass index and smoking. Additionally, we performed lag time analyses and corrected HRs for regression dilution bias.

Results Significant relationships of cholesterol with cancer incidence were all inverse including liver cancer ( $\mathrm{HR}=0.62$; 95\% CI 0.42 to $0.90)$ in males and cancers of the liver $(0.62 ; 0.49$ to 0.78$)$, gallbladder ( $0.62 ; 0.44$ to 0.85$)$, breast $(0.90 ; 0.85$ to 0.94$)$, and cancers of the lymph and haematopoietic tissue $(0.85 ; 0.76$ to 0.93$)$ in females. In lag analyses excluding cancer events occurring up to 5 years after cholesterol measurements, relationships persisted for liver, gallbladder and breast cancer.

Conclusions Total cholesterol was negatively associated with cancer risk at several sites. Lag time analyses suggested that these associations are only partially explained by reverse causation. 